\title{
The site of cholesterol oxidation impacts its localisation and domain formation in the neuronal plasma membrane
}

\section{Supporting Information}

Katie A. Wilson, Lily Wang, and Megan L. O'Mara*

Research School of Chemistry, College of Science, The Australian National University, Canberra, ACT, 2601, Australia

* Corresponding author

\section{Table of Contents}

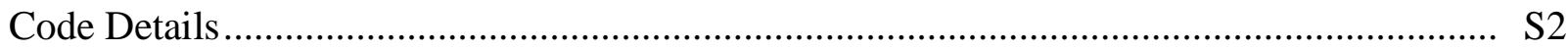

Calculation of local bilayer normal............................................................................ S2

Calculation of membrane thickness ....................................................................... S2

Contact fraction analysis for lipid domain formation ............................................... S2

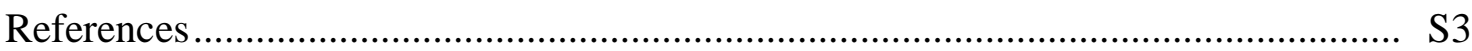

Table S1. Lipid composition of each system studied in the present work............................. S4

Figure S1 Line drawing of cholesterol, ring-oxidized cholesterol and tail-oxidized cholesterol with the coarse grain bead mapping .............................................................................. S6

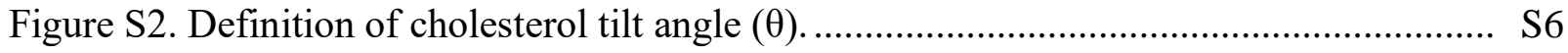

Figure S3. Leaflet content of cholesterol and oxidized cholesterol localization over time ..... S7

Figure S4. Membrane thickness over time. ....................................................................... S7

Figure S5. Snapshot of the water permeation. ................................................................... S8

Figure S6. Area per lipid of lipid classes separated by headgroup ......................................... S9

Figure S7. Mean lateral self-diffusivity for lipid classes separated by headgroup...................S10

Figure S8. Mean curvature and snapshot of the intracellular leaflet .........................................S11

Figure S9. Diagram of how the bilayer local normal is obtained. ...........................................S12 


\section{Code Details}

\section{Calculation of local bilayer normal}

The extracellular and intracellular leaflets of the membrane were first identified using the algorithm in Lipyds published in Wilson et al. (2021). ${ }^{1}$ As multiple headgroups may be selected for each lipid (e.g., lipids may contain both a PO4 and a GL1 bead), coordinates were averaged for each residue. These coordinates were then used to construct triangular meshes in PyVista ${ }^{2}$ using a 2D Delaunay triangulation (Figure S9). Periodic boundary conditions were accounted for by augmenting coordinates on each face of the cell, within $30 \AA$ (Figure S9b and S9c). An interleaflet mesh was then constructed using the mid-points of the upper and lower leaflets (Figure S9b). Specifically, the local normal was first computed for each leaflet. The distance to the other leaflet was calculated along this normal. The mid-point was deemed to lie on the local normal, at half the distance. Once the middle, inter-leaflet mesh surface was constructed, normals were calculated for each point using PyVista, which averages the local normals for each triangle. To ensure consistent signs of the local normal vector, the local normals were oriented to the global normal, which was the z-axis.

We note that this method differs from that of FATSLiM, another popular library for membrane analysis. Where FATSLiM estimates the local bilayer normal using a plane fit with principal component analysis to neighbouring lipids within a cutoff distance, this method determines the normal for each triangular face of a mesh and averages them at shared points. The approach used in this paper reflects smaller, higher-resolution changes in surface normals.

\section{Calculation of membrane thickness}

Leaflets were identified and an inter-leaflet mesh was constructed using the method described above. For each point of the inter-leaflet mesh, the local normal was calculated using PyVista. The distance to each leaflet was calculated along the local normal using ray tracing to draw a line to the point of intersection. The thickness $d_{i}$ for point $i$ was the sum of the per-leaflet distance. The thickness per frame $d$ of the membrane was the average of all points, to mitigate potential outlier values.

\section{Contact fraction analysis for lipid domain formation}

Lipid domain formation was interpreted from the contact fraction ratio. The contact fraction ratios of lipids to each other were carried out per-leaflet, using the leaflet identifying algorithm in Lipyds published in Wilson et al. (2021). ${ }^{l}$ The expected number of lipid-to-lipid contacts in a leaflet given complete mixing was calculated as the product of their proportions. In a leaflet comprising $\mathrm{N}$ lipids with $n_{A}$ molecules of lipid group A, the proportion of $\mathrm{A}$ is:

$$
\operatorname{Pr}(A)=\frac{n_{A}}{N}
$$


The expected number of contacts between lipid group A and lipid group B given complete mixing is:

$$
\operatorname{Pr}(A \cap B)=\operatorname{Pr}(A) \operatorname{Pr}(B)
$$

The contact fraction $(\mathrm{CF})$ ratio was calculated as the ratio of observed contacts between $\mathrm{A}$ and $\mathrm{B}$ to the expected number of contacts. That is, where $c_{A B}$ represents the number of contacts between lipids in group $\mathrm{A}$ and lipids in group $\mathrm{B}$, and where $\mathrm{C}$ represents the total number of contacts between lipids:

$$
C F_{A B}=\frac{c_{A B}}{C} \div \operatorname{Pr}(A \cap B)
$$

\section{References}

1. Wilson, K. A., Wang, L., Lin, Y. C., and O'Mara, M. L. (2021) Investigating the lipid fingerprint of SLC6 neurotransmitter transporters: a comparison of dDAT, hDAT, hSERT, and GlyT2, BBA Advances, 100010.

2. Sullivan, C., and Kaszynski, A. (2019) PyVista: 3D plotting and mesh analysis through a streamlined interface for the Visualization Toolkit (VTK), Journal of Open Source Software 4, 1450 . 
Table S1. Lipid composition of each system studied in the present work.

\begin{tabular}{|c|c|c|c|c|c|}
\hline Lipid & Class & Leaflet & $\begin{array}{l}\text { Neuronal } \\
\text { membrane }\end{array}$ & $\begin{array}{l}\text { Neuronal membrane } \\
\text { with } 10 \% \text { ring- } \\
\text { oxidized } \mathrm{CHOL}\end{array}$ & $\begin{array}{l}\text { Neuronal membrane } \\
\text { with } 10 \% \text { tail-oxidized } \\
\mathrm{CHOL}\end{array}$ \\
\hline DPCE & CER & extracellular & 8 & 8 & 8 \\
\hline DBCE & CER & extracellular & 2 & 2 & 2 \\
\hline PNCE & CER & extracellular & 2 & 2 & 2 \\
\hline $\mathrm{CHOL}$ & $\mathrm{CHOL}$ & extracellular & 898 & 695 & 695 \\
\hline OCLR & $\mathrm{CHOL}$ & extracellular & 0 & 202 & 0 \\
\hline CLRO & $\mathrm{CHOL}$ & extracellular & 0 & 0 & 202 \\
\hline PADG & DAG & extracellular & 6 & 6 & 6 \\
\hline PODG & DAG & extracellular & 2 & 2 & 2 \\
\hline DPG1 & GS & extracellular & 18 & 18 & 18 \\
\hline DPG3 & GS & extracellular & 18 & 18 & 18 \\
\hline DBG1 & GS & extracellular & 4 & 4 & 4 \\
\hline PNG1 & GS & extracellular & 4 & 4 & 4 \\
\hline DBG3 & GS & extracellular & 4 & 4 & 4 \\
\hline PNG3 & GS & extracellular & 4 & 4 & 4 \\
\hline POG1 & GS & extracellular & 2 & 2 & 2 \\
\hline POG3 & GS & extracellular & 2 & 2 & 2 \\
\hline DPGS & GS & extracellular & 99 & 99 & 99 \\
\hline PNGS & GS & extracellular & 22 & 22 & 22 \\
\hline DBGS & GS & extracellular & 18 & 18 & 18 \\
\hline POGS & GS & extracellular & 12 & 12 & 12 \\
\hline PPC & LPC & extracellular & 4 & 4 & 4 \\
\hline IPC & LPC & extracellular & 2 & 2 & 2 \\
\hline PPE & LPE & extracellular & 2 & 2 & 2 \\
\hline IPE & LPE & extracellular & 2 & 2 & 2 \\
\hline POPC & PC & extracellular & 175 & 175 & 175 \\
\hline DPPC & PC & extracellular & 107 & 107 & 107 \\
\hline PAPC & PC & extracellular & 93 & 93 & 93 \\
\hline DOPC & PC & extracellular & 44 & 44 & 44 \\
\hline PUPC & PC & extracellular & 34 & 34 & 34 \\
\hline PFPC & PC & extracellular & 12 & 12 & 12 \\
\hline OIPC & PC & extracellular & 12 & 12 & 12 \\
\hline OUPC & PC & extracellular & 8 & 8 & 8 \\
\hline PUPE & PE & extracellular & 101 & 101 & 101 \\
\hline PAPE & PE & extracellular & 62 & 62 & 62 \\
\hline POPE & $\mathrm{PE}$ & extracellular & 26 & 26 & 26 \\
\hline OAPE & PE & extracellular & 14 & 14 & 14 \\
\hline OUPE & PE & extracellular & 14 & 14 & 14 \\
\hline OIPE & PE & extracellular & 2 & 2 & 2 \\
\hline DPSM & SM & extracellular & 117 & 117 & 117 \\
\hline PNSM & SM & extracellular & 26 & 26 & 26 \\
\hline PBSM & SM & extracellular & 22 & 22 & 22 \\
\hline POSM & SM & extracellular & 14 & 14 & 14 \\
\hline DPCE & CER & intracellular & 8 & 8 & 8 \\
\hline DBCE & CER & intracellular & 2 & 2 & 2 \\
\hline PNCE & CER & intracellular & 2 & 2 & 2 \\
\hline $\mathrm{CHOL}$ & $\mathrm{CHOL}$ & intracellular & 902 & 699 & 699 \\
\hline OCLR & $\mathrm{CHOL}$ & intracellular & 0 & 202 & 0 \\
\hline CLRO & $\mathrm{CHOL}$ & extracellular & 0 & 0 & 202 \\
\hline PADG & DAG & intracellular & 6 & 6 & 6 \\
\hline PODG & DAG & intracellular & 2 & 2 & 2 \\
\hline PPC & LPC & intracellular & 2 & 2 & 2 \\
\hline IPC & LPC & intracellular & 2 & 2 & 2 \\
\hline IPE & LPE & intracellular & 4 & 4 & 4 \\
\hline
\end{tabular}




\begin{tabular}{|c|c|c|c|c|c|}
\hline PPE & LPE & intracellular & 2 & 2 & 2 \\
\hline PAPA & PA & intracellular & 6 & 6 & 6 \\
\hline POPA & PA & intracellular & 2 & 2 & 2 \\
\hline POPC & PC & intracellular & 99 & 99 & 99 \\
\hline DPPC & PC & intracellular & 60 & 60 & 60 \\
\hline PAPC & PC & intracellular & 52 & 52 & 52 \\
\hline DOPC & PC & intracellular & 24 & 24 & 24 \\
\hline PUPC & PC & intracellular & 20 & 20 & 20 \\
\hline PFPC & PC & intracellular & 6 & 6 & 6 \\
\hline OIPC & PC & intracellular & 6 & 6 & 6 \\
\hline OUPC & PC & intracellular & 4 & 4 & 4 \\
\hline PUPE & PE & intracellular & 196 & 196 & 196 \\
\hline PAPE & $\mathrm{PE}$ & intracellular & 123 & 123 & 123 \\
\hline POPE & $\mathrm{PE}$ & intracellular & 50 & 50 & 50 \\
\hline OUPE & $\mathrm{PE}$ & intracellular & 28 & 28 & 28 \\
\hline OAPE & $\mathrm{PE}$ & intracellular & 26 & 26 & 26 \\
\hline OIPE & $\mathrm{PE}$ & intracellular & 6 & 6 & 6 \\
\hline PUPI & $\mathrm{PI}$ & intracellular & 40 & 40 & 40 \\
\hline POPI & $\mathrm{PI}$ & intracellular & 26 & 26 & 26 \\
\hline PAPI & $\mathrm{PI}$ & intracellular & 26 & 26 & 26 \\
\hline PIPI & $\mathrm{PI}$ & intracellular & 10 & 10 & 10 \\
\hline PAP1 & $\mathrm{PI}$ & intracellular & 6 & 6 & 6 \\
\hline PAP2 & $\mathrm{PI}$ & intracellular & 6 & 6 & 6 \\
\hline PAP3 & $\mathrm{PI}$ & intracellular & 6 & 6 & 6 \\
\hline POP1 & $\mathrm{PI}$ & intracellular & 4 & 4 & 4 \\
\hline POP2 & $\mathrm{PI}$ & intracellular & 4 & 4 & 4 \\
\hline POP3 & $\mathrm{PI}$ & intracellular & 4 & 4 & 4 \\
\hline PUPS & PS & intracellular & 68 & 68 & 68 \\
\hline PAPS & PS & intracellular & 56 & 56 & 56 \\
\hline POPS & PS & intracellular & 50 & 50 & 50 \\
\hline OUPS & PS & intracellular & 14 & 14 & 14 \\
\hline DPPS & PS & intracellular & 10 & 10 & 10 \\
\hline DPSM & SM & intracellular & 30 & 30 & 30 \\
\hline PNSM & SM & intracellular & 6 & 6 & 6 \\
\hline PBSM & SM & intracellular & 6 & 6 & 6 \\
\hline POSM & SM & intracellular & 4 & 4 & 4 \\
\hline
\end{tabular}


a)
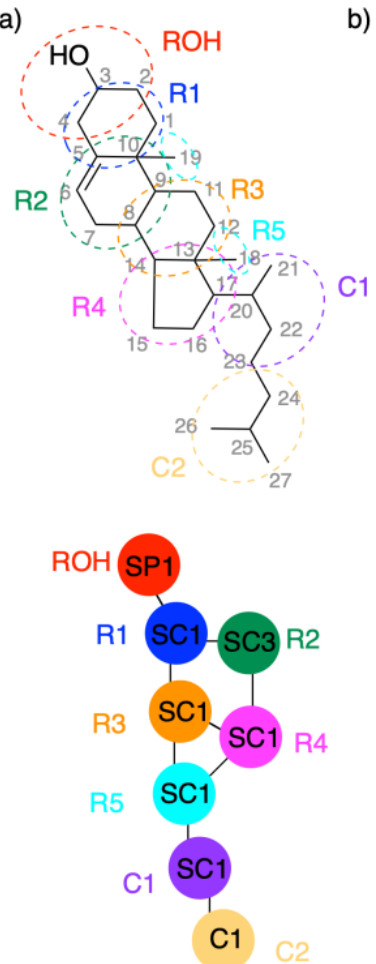

b)
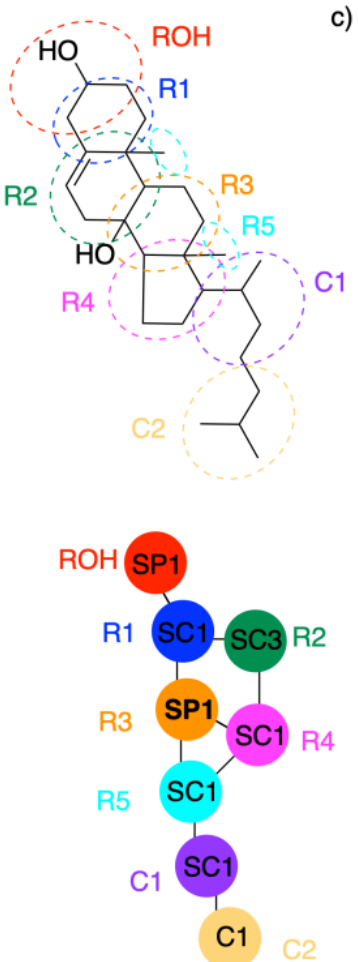

c)
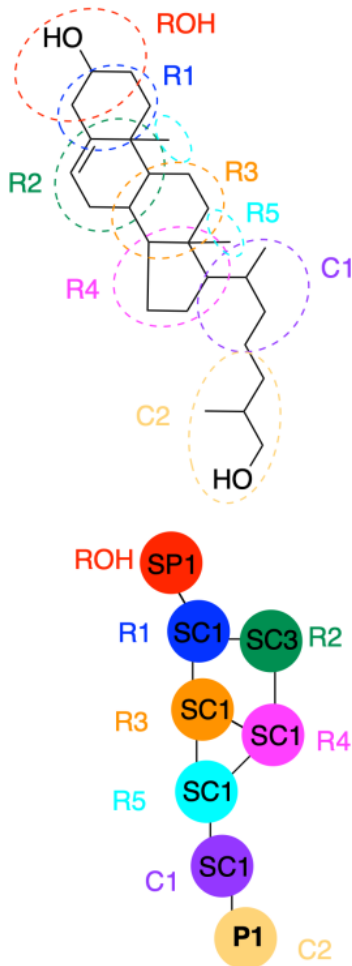

Figure S1. Line drawing of a) cholesterol, b) ring-oxidized cholesterol and c) tail-oxidized cholesterol with the coarse grain bead mapping shown with circles (top) and Martini representation (bottom). Note that the chemical structures shown for ring-oxidized and tail-oxidized cholesterol are examples and multiple oxidized species can be represented by the coarse grain representations for ring-oxidized and tail-oxidized cholesterol. In ring-oxidized cholesterol, the R3 bead contains the oxidation site and is assigned the SP1 Martini bead type. In tail-oxidized cholesterol, the C2 bead contains the oxidation site and is assigned the P1 Martini bead type.

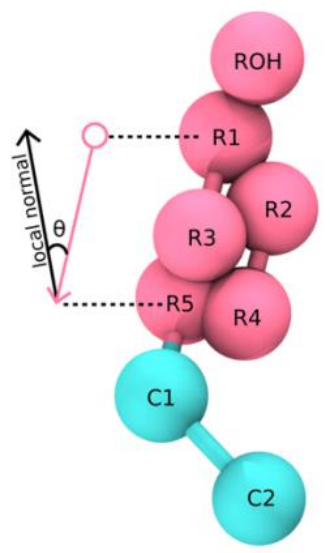

Figure S2. Definition of cholesterol tilt angle $(\theta)$. 

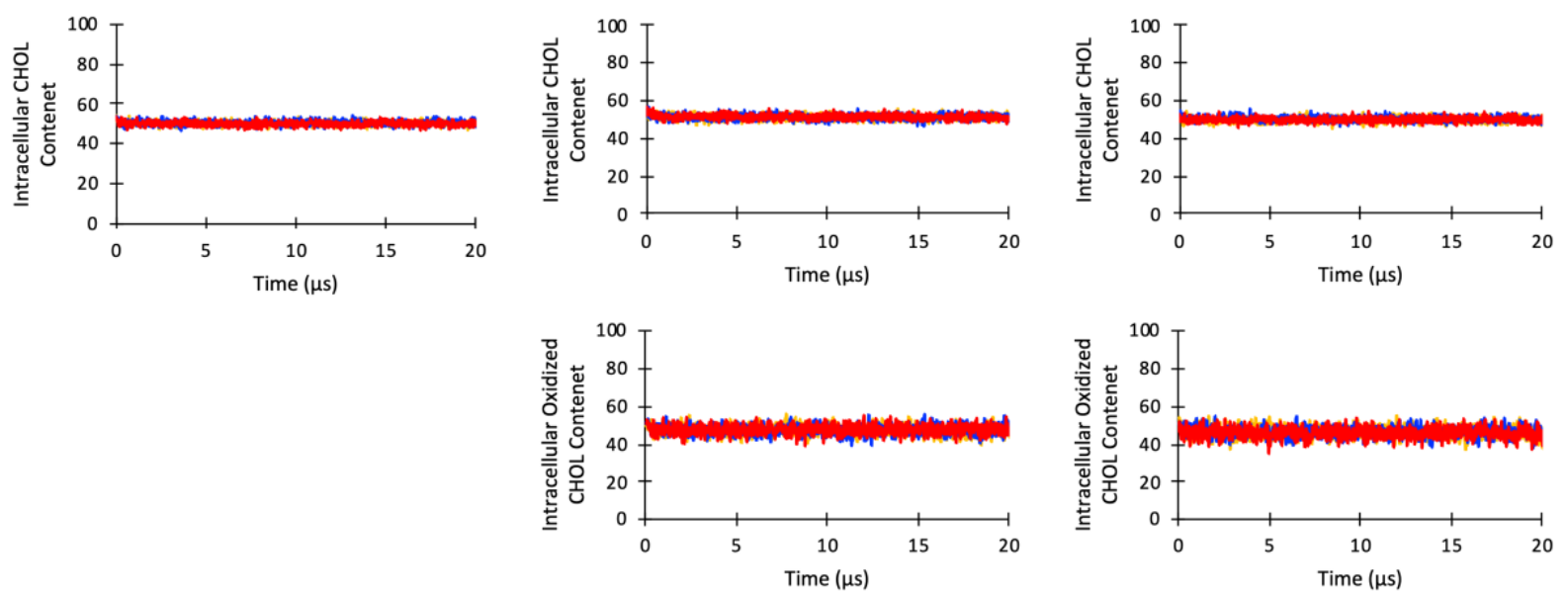

Figure S3. Intracellular leaflet content of cholesterol (top) and oxidized cholesterol (bottom) in the localization over time in the neuronal membrane (left), neuronal membrane with $10 \%$ ringoxidized cholesterol (middle) and neuronal membrane with $10 \%$ tail-oxidized cholesterol (right) for replicate 1 (yellow), replicate 2 (blue) and replicate 3 (red).

a)

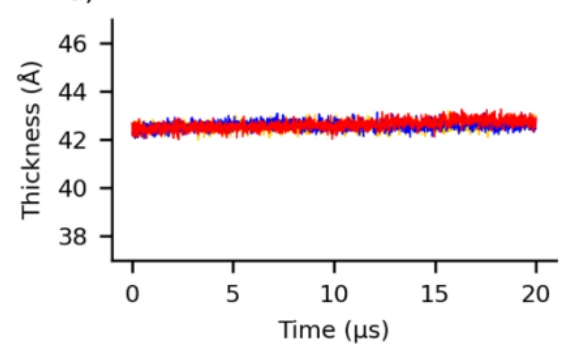

b)

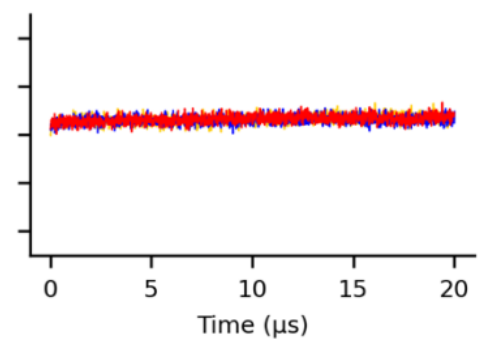

c)

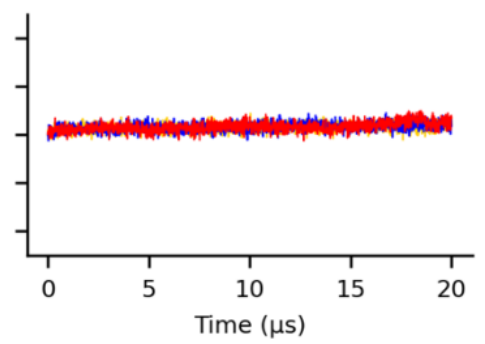

Figure S4. Membrane thickness over time for the neuronal membrane (a), neuronal membrane with $10 \%$ ring-oxidized cholesterol (b) and neuronal membrane with $10 \%$ tail-oxidized cholesterol (c) for replicate 1 (yellow), replicate 2 (blue) and replicate 3 (red). 

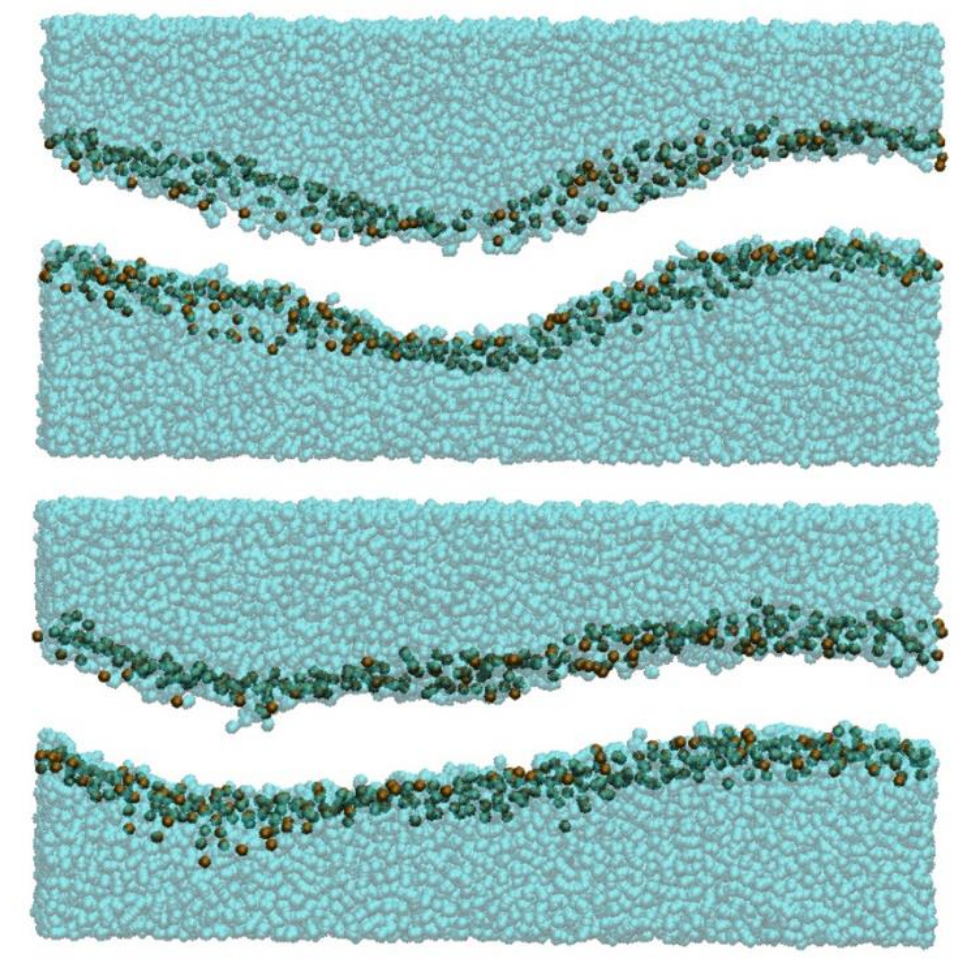

Figure S5. Snapshot of the water permeation through the neuronal membrane with $10 \%$ ringoxidized cholesterol (top) or $10 \%$ tail-oxidized cholesterol (bottom), taken from the final frame of the coarse-grained simulation. To allow a cross-section view of the water penetration, the image was cropped to a depth of $100 \AA$ in the y-direction. The MARTINI polarizable water is shown in transparent cyan spacefill and the lipid phosphate groups are shown in ochre spacefill. 
a)

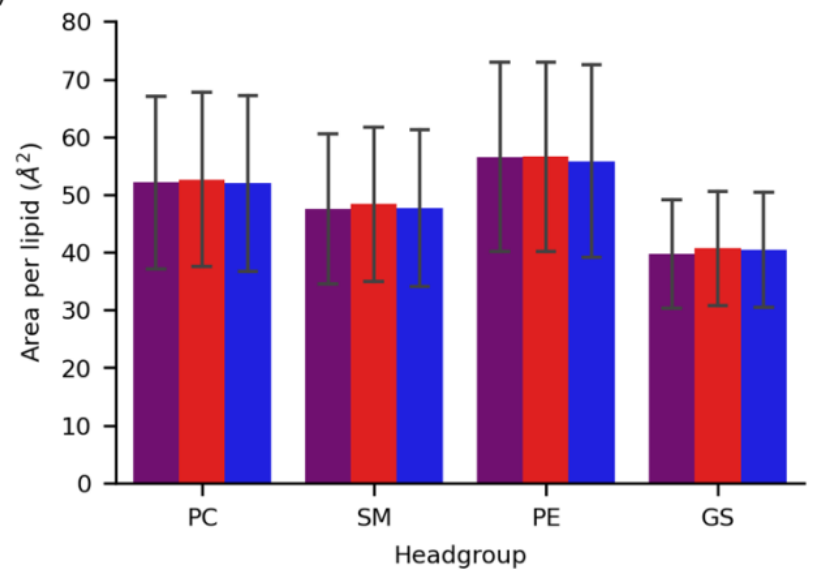

b)

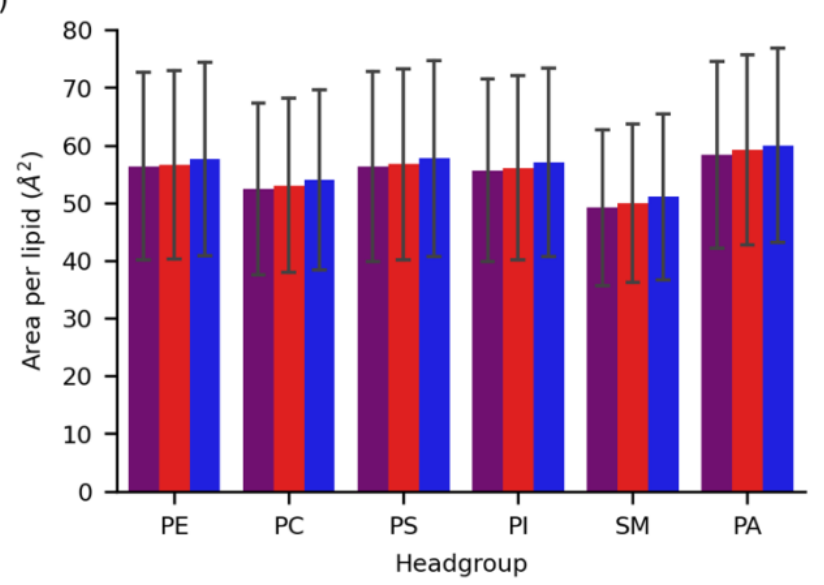

Figure S6. Area per lipid of membrane lipid classes separated by headgroup in the extracellular (a) or intracellular (b) leaflets for the neuronal membrane (purple), neuronal membrane with $10 \%$ ring-oxidized cholesterol (red) and neuronal membrane with 10\% tail-oxidized cholesterol (blue). 

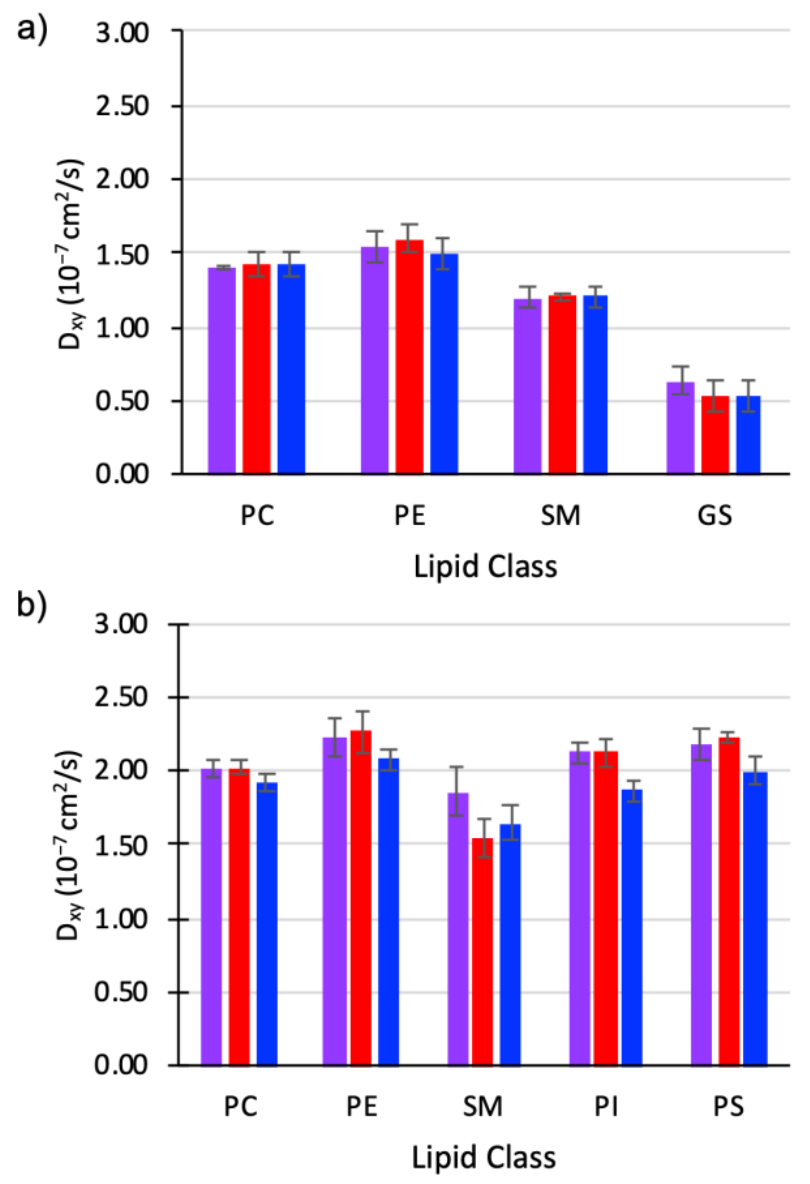

Figure S7. Mean lateral self-diffusivity for membrane lipid classes separated by headgroup in the a) extracellular or b) intracellular leaflets for the neuronal membrane (purple), neuronal membrane with $10 \%$ ring-oxidized cholesterol (red) and neuronal membrane with $10 \%$ tail-oxidized cholesterol (blue). Error bars represent the standard error of the mean. 

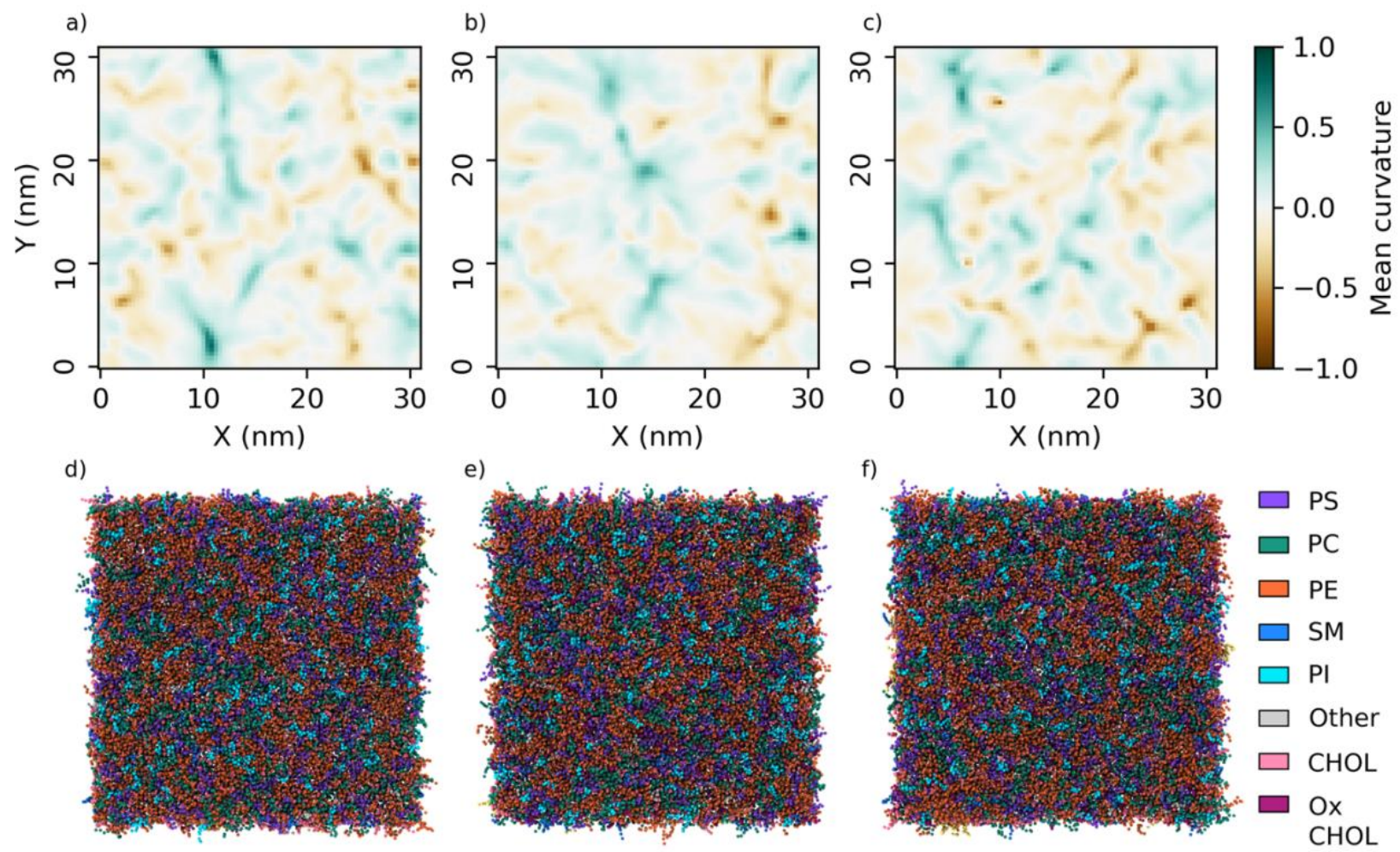

Figure S8. Mean curvature (a, b, c) and snapshot of the intracellular leaflet (d, e, f) of the neuronal membrane (a, d), neuronal membrane with $10 \%$ ring-oxidized cholesterol $(b, e)$ and neuronal membrane with $10 \%$ tail-oxidized cholesterol (c, f). The first replicate for each system is shown, at the last frame. 
a)

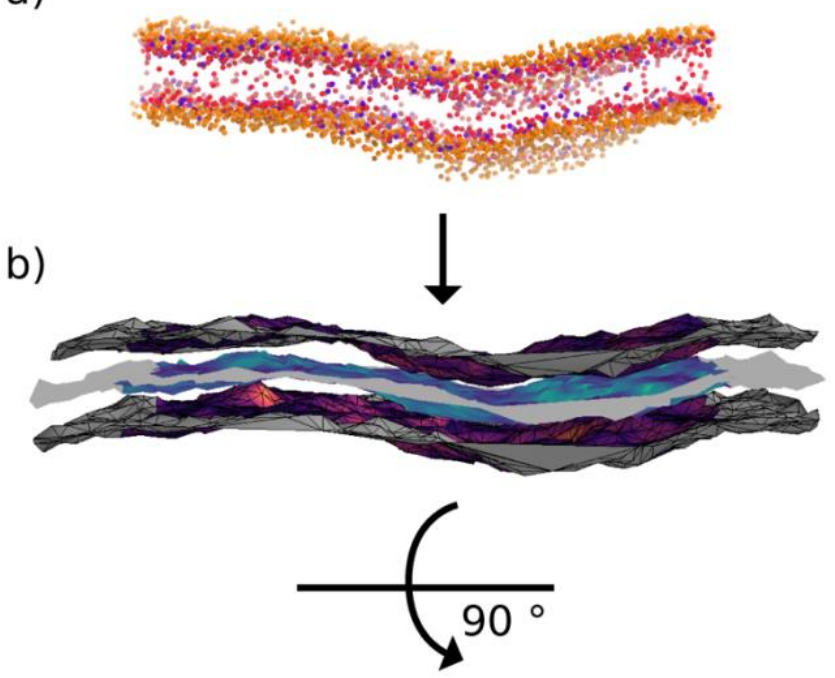

C)

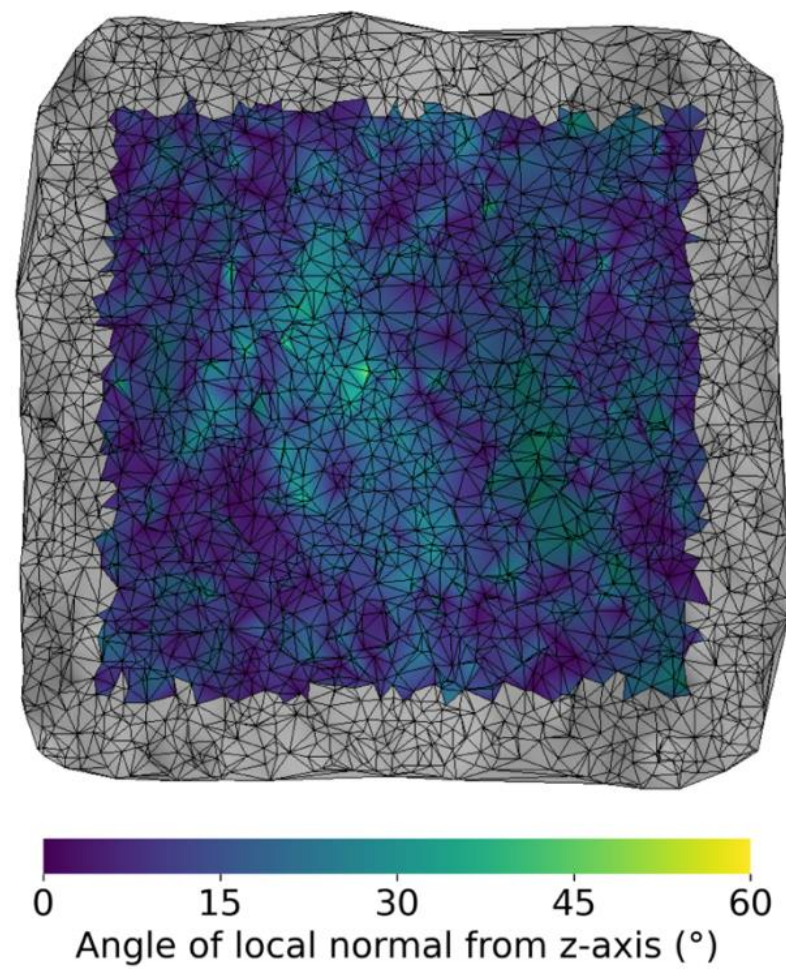

Figure S9. Diagram of how the bilayer local normal is obtained from triangular meshes of the membrane. a) the headgroups of an example frame of the membrane with $10 \%$ tail-oxidised cholesterol. Yellow: phospholipid head groups. Red: cholesterol headgroup. Purple: hydroxycholesterol headgroup. b) diagram of the triangular meshes of the upper and lower leaflets, as well as the projected middle. The grey portions of the membrane are augmented coordinates to account for periodic boundary conditions. c) a top-down view of the middle, inter-leaflet triangular mesh. The coordinates of the membrane are colored by the angle of the local normal from the global z-axis. The grey portions of the membrane are augmented coordinates to account for periodic boundary conditions. 